\title{
Optimization of continuous-flow diphenyldiazomethane synthesis: an integrated undergraduate chemistry experiment
}

\author{
Luuk T. C. G. van Summeren ${ }^{1}$ • Jan Gerretzen ${ }^{2}$ • Floris P. J. T. Rutjes ${ }^{1,3} \cdot$ Tom G. Bloemberg $^{1,3}$ (D)
}

Received: 30 May 2020 / Accepted: 5 August 2020 / Published online: 8 September 2020

(C) The Author(s) 2020

\begin{abstract}
We present a challenging flow-chemistry experiment concerning the synthesis of diphenyldiazomethane using the OmuraSharma-Swern oxidation, that we have developed and used in our second-year undergraduate lab classes over the past seven years. The experiment integrates a number of different aspects and concepts of chemistry that are traditionally taught as separate subjects in undergraduate chemical education: organic synthesis, quantitative chemical analysis, design of experiments, optimization, statistical modelling, computer programming and continuous-flow processes.
\end{abstract}

Keywords Diphenyldiazomethane $\cdot$ Chemical education $\cdot$ Flow chemistry $\cdot$ Experimental design $\cdot$ DoE

\section{Introduction}

Although continuous-flow processes are a mainstay of industrial bulk chemistry, the advent of microreactors has constituted a paradigm change in laboratory-scale synthetic chemistry. As such, it is important that this is also reflected in chemical education programmes [1]. At the same time, by its nature, flow chemistry also offers opportunities for integrating parts of chemical education in lab courses that are otherwise hard to incorporate.

\section{Highlights}

- A challenging undergraduate flow chemistry experiment is presented

- A new manner of producing diazo compounds in flow chemistry is presented

- Ideas for extension of the experiment are provided

Electronic supplementary material The online version of this article (https://doi.org/10.1007/s41981-020-00107-4) contains supplementary material, which is available to authorized users.

Tom G. Bloemberg

t.bloemberg@science.ru.nl

1 Educational Institute for Molecular Sciences, Radboud University, Heyendaalsweg 135, 6525, AJ Nijmegen, The Netherlands

2 Nouryon Chemicals B.V., Integrated Supply Chain, Advanced Analytics and Control, Zutphenseweg 10, 7418, AJ Deventer, The Netherlands

3 Institute for Molecules and Materials, Radboud University, Heyendaalsweg 135, 6525, AJ Nijmegen, The Netherlands
We have been teaching flow chemistry in our lab courses since 2011, when we acquired two b330 flow start education setups from FutureChemistry, a spin-off company from our university specializing in flow chemistry. From the start, the Omura-Sharma-Swern oxidation has been the reaction that we use to make students acquainted with flow chemistry. The first two years, we provided students with an application note provided by FutureChemistry, based on [2] and describing the straightforward synthesis of cinnamyl aldehyde by oxidation of cinnamyl alcohol.

In 2013, we realized that this same reaction could also be used to convert benzophenone hydrazone (1, Fig. 1) to diphenyldiazomethane (2) [3, 4], This reaction is more appealing from a didactic viewpoint, for several reasons:

- the product is a relatively stable diazo compound: a chemically interesting type of compound [5,6], attractive to students, but not frequently encountered in undergraduate education;

- the mechanism of the formation of the product (Fig. 2B) differs sufficiently from that of the commonly encountered aldehyde or ketone analogues (Fig. 2A) to be challenging for students to derive;

- last but not least, the product has an intense purple-red color, permitting observation of its formation by eye as well as allowing quantification by Vis spectroscopy, for which equipment is readily available in most undergraduate laboratories. 


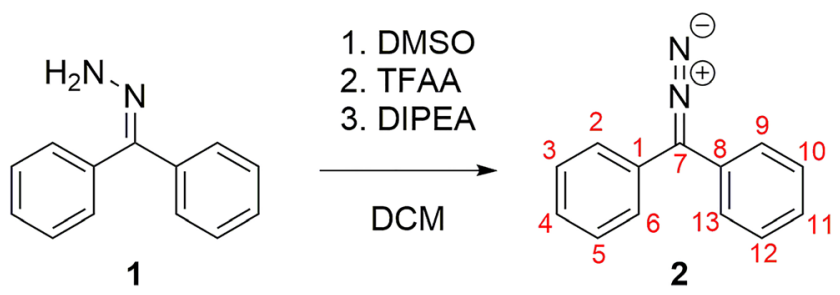

Fig. 1 The synthesis of diphenyldiazomethane 2 via the OmuraSharmaSwern oxidation of benzophenone hydrazone 1. DMSO: dimethyl sulfoxide; TFAA: trifluoroacetic anhydride; DIPEA: N,Ndiisopropylethylamine; DCM: dichloromethane.

We proceeded to investigate whether the synthesis of $\mathbf{2}$ could indeed also be performed in continuous flow at room temperature and to our delight this proved to be the case, even with our relatively simple flow chemistry setups. We then formulated a two-day student project in which students not only synthesize $\mathbf{2}$, but actually perform a limited optimization of two reaction parameters by means of a formal experimental design, integrating the theory they have previously obtained about organic chemistry, analytical chemistry, design of experiments (DoE), statistics and programming with the new concept of continuous flow processes.

Since then, the synthesis of $\mathbf{2}$ in flow has been one of the two recurring instances of flow chemistry in our undergraduate synthetic lab courses; the other being the routine synthesis of ethyl diazoacetate in flow [7] on an as-needed basis for use in the synthesis of a bicyclononyl derivative [8].

In this paper, we will discuss the optimization of the synthesis of $\mathbf{2}$ both from an experimental and from a chemical education viewpoint. A basic student manual for the experiment is provided as part of the Supporting Information.

\section{Educational / didactical value}

Different theories about learning exist and although we will not go into much detail in the current paper, it is important to mention that the integration [9] of knowledge is generally accepted to be one of the 'highest' forms of knowledge.
These forms of knowledge build upon, or are converted from, 'lower' forms of knowledge such as remembering (as in rote memorization) and understanding [10]. Another influential idea is that learning something new is facilitated by being able to connect it to prior knowledge [11], which therefore needs to be 'activated' at the proper moment. This activation in turn can be effectively achieved by applying knowledge, such as when performing an experiment in a lab course.

The diphenyldiazomethane experiment is explicitly aimed at learning something new (continuous-flow processes) by connecting it to prior knowledge (in particular classic organic synthesis via batch processes) whilst at the same time integrating and applying prior knowledge about chemical subjects and concepts such as organic chemistry, quantitative analytical chemistry, kinetics, optimization, experimental design, statistics and computer programming. With the exception of a minority of students explicitly pursuing more advanced analytical or theoretical chemical education, students are otherwise not challenged to activate and thereby maintain let alone upgrade - their knowledge about many of these subjects and concepts until they start their professional careers.

\section{Moffatt oxidations}

Following the introduction of 'activated DMSO' oxidations at the end of the 1950s, the direct oxidations of primary and secondary alcohols to the corresponding aldehydes and ketones by Moffatt-style DMSO-based activators at the beginning of the 1960s have become a standard tool in synthetic laboratories [12-17]. The general procedure involves activating DMSO by having it react with an 'activator', an electrophile that is attacked by a lone pair on the oxygen atom of DMSO. Two of the activators leading to the most reactive activated DMSO species are oxalyl chloride and trifluoroacetic anhydride, both introduced by Swern and coworkers [18-22].

There can be a cruel sense of humor in scientific progress: after Albright et al. in the 1960's had dismissed the use of trifluoroacetic anhydride as an activator [23, 24], because it
Fig. 2 A: The general mechanism for the Moffatt oxidation of an alcohol by activated DMSO. B: Proposed mechanism for the formation of $\mathbf{2}$ from $\mathbf{1}$ by activated DMSO. The intramolecular transfer of a proton in the third step takes place in a fourmembered ring, while in the general mechanism a five-membered ring is involved here. It is conceivable that in this case, the amine proton is transferred directly to the base. a
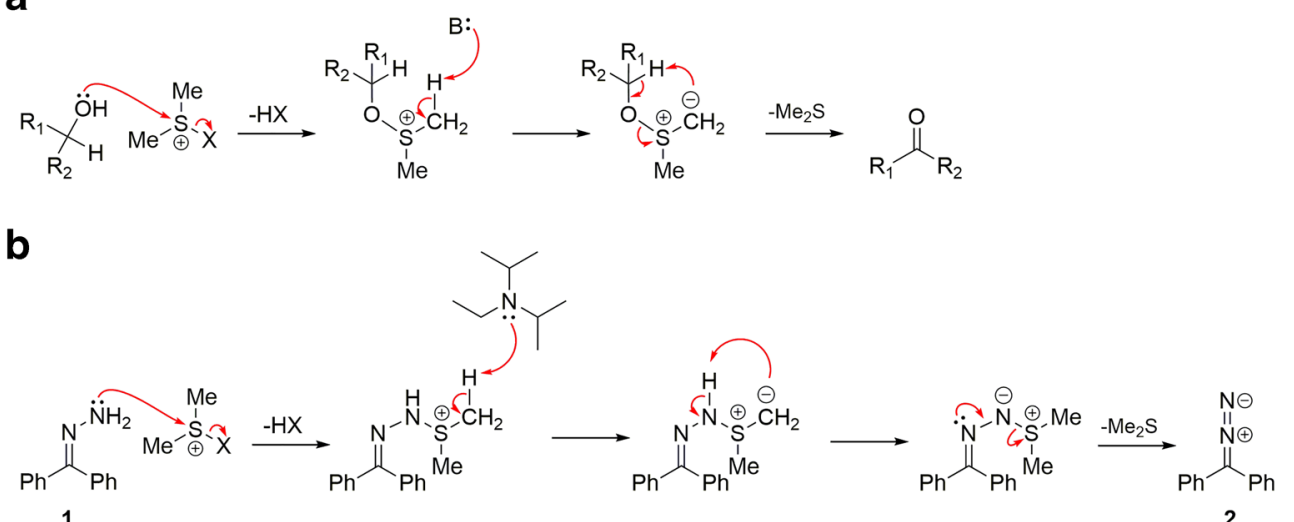
was useless at room temperature, Swern and coworkers, in the 1970 's proved that it actually is a very good activator at low temperatures $[21,22,25]$ and with the advent of flow chemistry, the use of trifluoroacetic anhydride as an activator for DMSO at room temperature has been proven possible after all [2, 26, 27].

Contrary to their use in the oxidization of alcohols, Moffatt oxidations are far less widely used for the analogous conversion of amines into the corresponding imines [28-31], probably not because of inherent issues, but because the formation of an imine from an amine is simply less frequently required than the opposite pathway, as in reductive amination. Whereas the mechanism of imine formation starting from a secondary amine is probably analogous to that of carbonyl formation starting from an alcohol [32, Fig. 2A), it is not immediately obvious that it is also possible to convert a hydrazone into a diazo group, since in that case, two hydrogen atoms need to be abstracted from the same nitrogen (Fig. 2B). Not surprisingly therefore, the discovery of this possibility has been described as serendipitous and provides an extension of the set of available ways to produce diazo compounds. As such, the current paper represents a further extension to the ways available for producing diazo compounds in a continuous-flow setup [7, 33-35]. The proposed mechanism is depicted in Fig. 2B.

\section{The experiment}

Over the years, our undergraduate experiment has seen many small changes. In its current simplest version, students are tasked to prepare three $20 \mathrm{~mL}$ solutions of trifluoroacetic acid (TFAA, $0.4 \mathrm{M}$ ), N,N-diisopropylethylamine (DIPEA, $1.435 \mathrm{M}$ ) and, analogous to references 2 and 27, a combination of dimethyl sulfoxide (DMSO, 0.6 M) and $1(0.2 \mathrm{M})$, all in dichloromethane (DCM). The TFAA and 1/DMSO solutions are then taken up in two $5 \mathrm{~mL}$ glass syringes, while the DIPEA solution is taken up into a $1 \mathrm{~mL}$ glass syringe. The reason for this difference is that the required flow rates for the DIPEA solution are generally quite low and with a large syringe, the step motor of the pump would have to rotate so slowly that the actual flow rate might become exceedingly inaccurate and/or the flow might become discontinuous.

The syringes are placed in three syringe pumps and connected via short $(\sim 10 \mathrm{~cm})$ inlet tubing to the $1 \mu \mathrm{L}$ microreactor (Fig. 3) which is placed in a reactor holder on top of a Peltier element set to cool down to $10{ }^{\circ} \mathrm{C}$. In the student experiments, the temperature is kept constant, since from previous experience, we know that its effect is roughly inversely related to the residence time of the reactants in the microreactor. Of these two, therefore, only the residence time is used as a factor in the subsequent optimization procedure. A microtube is

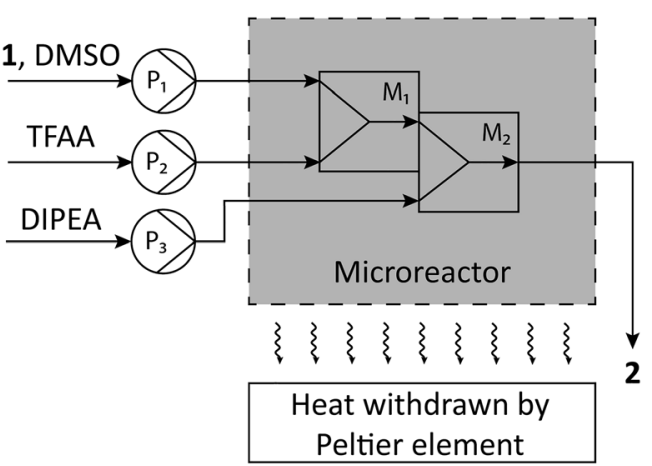

Fig. 3 Microreactor setup: the 1 / DMSO and TFAA solutions are combined and mixed in mixing channel M1 which has an internal volume of 1 $\mu \mathrm{L}$. At M2 the DIPEA solution is added, after which the reaction mixture leaves the microreactor.

connected to the microreactor outlet and stuck through a hole in the plastic cap of a standard $10 \mathrm{~mL}$ disposable glass vial.

After the setup has been checked for correctness and safety by a teacher or TA, it is suggested to students to first make sure that all inlet tubes are filled with their respective liquids by temporarily applying relatively high flow rates. Then, the flow rates are set to the 'standard settings' provided to them and their first synthesis of diphenyldiazomethane commences. After 5 to $10 \mathrm{~min}$, red droplets normally start to appear from the end of the outlet tubing [36]. When they have collected some of the effluent in a volume of DCM, the students proceed to use a UV-Vis spectrometer to measure the visible absorption spectrum of the solution to determine $\lambda_{\max }$, the wavelength of maximal absorbance.

Students then start the actual experimentation in which they are going to vary the different flow rates in order to optimize the conversion of $\mathbf{1}$ into $\mathbf{2}$ as a function of two factors: 1. the 'residence' time between mixing the TFAA and DMSO / 1 solutions and adding in the DIPEA, and 2. the ratio between TFAA and 1. Since 1 and DMSO are contained in the same solution, the ratio between TFAA and DMSO is varied at the same time, and in the same manner, so any effects due to the ratio TFAA/ $\mathbf{1}$ are confounded with (cannot be distinguished from) effects due to the TFAA/DMSO ratio.

In their statistics course, students have previously learned about Experimental Design or Design of Experiments (DoE), which describes ways to optimize a criterion as a function of a set of factors in the minimum number of experiments [37, 38]. Part of their preparation for the experiment is setting up an experimental design for the two factors. The basic option is to choose a full factorial design of two factors at two levels (Fig. 4). The number of experiments in this design is only four, but since students start with the standard settings as the center point to allow for curvature detection / estimation in the response surface, and also have to perform a number of replicates to assess if any observed effects are real or just due to random error, seven or eight experiments are normally the 


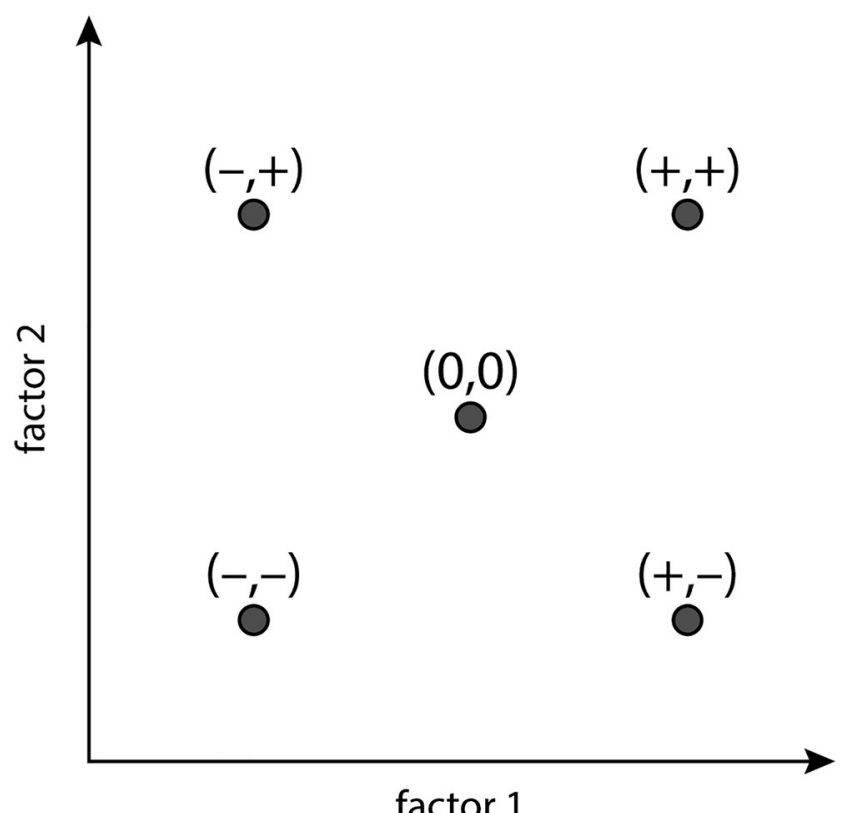

factor 1

Fig. 4 Schematic depiction of a full factorial design of two factors at two levels, a high (+) and a low (-) level. Each point corresponds to an experiment with the given factor settings (e.g. temperature, pressure, $\mathrm{pH}$, concentration of a reactant, etc.) in which the response (the quantity of interest, such as yield, ee, purity, etc.) is determined. The center point is not formally part of the full factorial design, but is often the starting point. It also allows for curvature detection of the response surface, which is vital if it is expected to have a maximum that is not an edge extreme.

minimum they should carry out. It is exactly here that one of the huge benefits of flow chemistry becomes apparent once again: imagine doing seven or eight batch experiments on a single day, let alone telling second-year undergraduate students that this is the bare minimum that you expect from them! Flow chemistry enables students to carry out multiple experiments, varying parameters in between, taking replicates and making mistakes and correcting for them. All these things are unimaginable for a classic synthesis experiment in the limited time frame of a single day.

For each combination of factor settings, students collect the red effluent from the microreactor in a volume of dichloromethane over the course of five to ten minutes. After collecting the product, students change the flow rates of the setup according to their experimental design (the order of which has preferably been randomized) and allow some time for equilibration of the system and removal of the effluent that is still in the outlet channel and corresponds to the previous factor settings. Meanwhile, they measure the absorbance of the diluted effluent on the UV-Vis spectrometer at $\lambda_{\max }$.

After they have carried out their experiments, students clean the setup and proceed to analyze their results, either by hand using direct effect estimation methods or by (linear) regression in a program such as Matlab, R or Microsoft Excel or by using a programming language such as Python [37].

\section{Results and discussion}

In an extension of the experiment, beyond the limited version for students, we have carried out a full characterization of the response surface of the relative conversion of $\mathbf{1}$ into $\mathbf{2}$ as a function of the two factors 'residence time' and 'TFAA/1 ratio' (confounded with TFAA/DMSO ratio).

The 'Vis' absorption spectrum of the product mixture shows a maximum around $\lambda_{\max }=521 \mathrm{~nm}$. Absorbance measurements to determine the relative conversion of $\mathbf{1}$ into $\mathbf{2}$ are carried out at that wavelength. In order to obtain good results, effluent was collected over some time to allow temporal variations in the conversion to average out. For each experiment, the effluent was collected in a glass vial over a precalculated time interval that was chosen such that the total amount of $\mathbf{1}$ that passed through the system was always equal. Afterwards, either $0.5 \mathrm{~mL}$ or $1.0 \mathrm{~mL}$ DCM was added. In this way, the volume of the colored liquid became large enough to fill a semi-micro cuvette, while at the same time, its absorbance became sufficiently low to stay below 1 and, hence, was in the regime where the Beer-Lambert law is valid.

The absorbance alone is not a good measure of the conversion, however, since - to a certain extent - increasing the amount of 1 by increasing flow rate 1 (Fig. 3) or decreasing the total volume of liquid by decreasing flows 2 and/or 3 may also result in higher concentrations of product in the effluent, even when relatively less $\mathbf{1}$ is converted into product. Therefore, the measured absorbances were normalized by dividing them by the theoretical concentration of $\mathbf{2}$ in case of total conversion. The remaining differences in the corrected absorbance are either due to an actual effect (i.e. an actual change in the conversion) or to factors beyond control (i.e. experimental error). The Microsoft Excel sheet with our bookkeeping and calculations is provided as Supporting Information.

Figure 5 shows two depictions of the response surface corresponding to the relative conversion of $\mathbf{1}$ into $\mathbf{2}$. Numerical results can be obtained from the spreadsheet in the Supporting Information. Some replicate measurements produced values that we suspected to be outliers. To reduce their influence in subsequent data fitting, we calculated the median values of the corrected absorbances and used these as input for the model fit. We also realized that the $[\mathrm{TFAA}] /[1]$ axis would be asymmetrical: starting from an equimolar situation (i.e. [TFAA]/ $[\mathbf{1}]=1$ ), and increasing [TFAA] by a factor of 2 leads to $[$ TFAA $] /[1]=2$, whereas decreasing it with a factor of 2 leads to $[$ TFAA $] /[1]=0.5$. To correct for this, we used the natural logarithm of $[\mathrm{TFAA}] /[\mathbf{1}]$ to render the axis symmetrical around 0 . This resulted in better fits (lower RMS) than for the asymmetrical situation. The data were fit to the model $z=a+b x+c y+d x y+e x^{2}+f y^{2}$, in which $z$ is the corrected absorbance, $x$ is the reaction time $t_{\text {residence }}$ (s) and $y$ is 


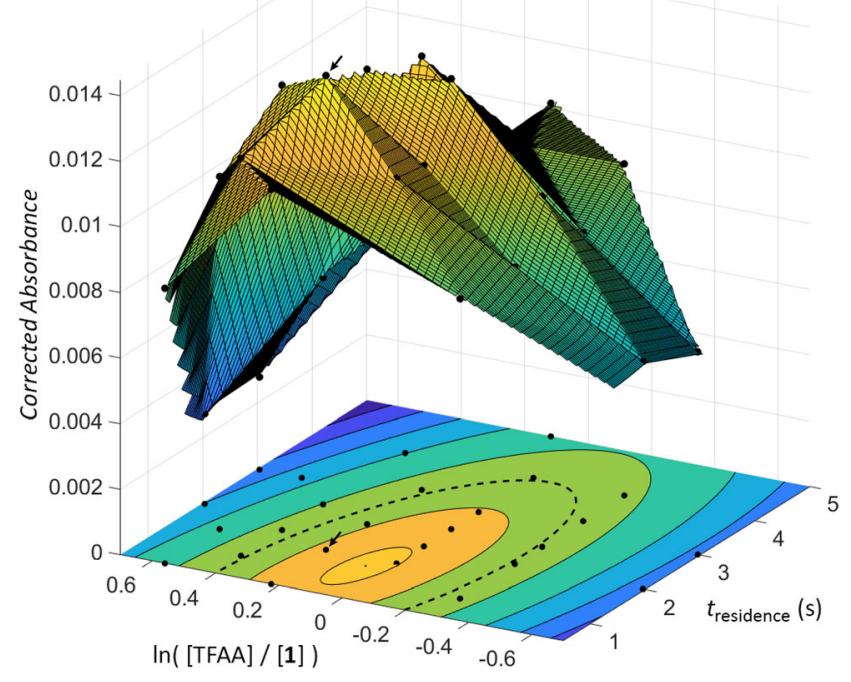

Fig. 5 Two depictions of the response surface of the corrected absorbance as a measure for relative conversion of 1 into 2 . The 3D surface simply 'connects the dots', which are the median values of replicate measurements of the corrected absorbance for different combinations of $t_{\text {residence }}$ and $\ln \frac{[\mathrm{TFAA}]}{[1]}$. The contour lines at the bottom show the fit of the quadratic model to the data. The distance between the contour lines corresponds to steps of 0.02 in the $\mathrm{z}$ direction, but the innermost contour line is added for extra detail at a distance of 0.006 from the previous one. The dashed contour line corresponds to a distance of 1 pooled standard deviation - calculated from all replicate measurements - below the maximum. The arrows indicate the point that yielded optimal experimental results.

$\ln \frac{[\text { TFAA }]}{[1]}$. The values obtained for the coefficients $a$ till $f$ can be found in the Supporting Information.

Although the fit of the quadratic model to the data is far from perfect, the addition of higher-order terms resulted in overfitting, whereas the exchange of the quadratic terms for higher order terms resulted in fits that were actually worse, although the location of the optimum hardly changed. This conforms to the observation made in [37] that in experimental optimization, models do not normally benefit from incorporating terms beyond quadratic ones. For the given concentrations and the set-up used, at a temperature of $10^{\circ} \mathrm{C}$, the model predicts the optimal combination of factors to be around $t_{\text {residence }}=1.75 \mathrm{~s}$ and $\ln \frac{[\mathrm{TFAA}]}{[1]}=0.065$. Experimentally, we obtained optimal results at $t_{\text {residence }}=1.5 \mathrm{~s}$ and $\ln \frac{[\mathrm{TFAA}]}{[1]}=0.2231$.

\section{Purification}

Although assessing the yield and purity of diazo compounds is notoriously difficult [3, 39], we attempted to purify an amount of continuous-flow product for further characterization by NMR and IR spectroscopy. The small amount of product was obtained as a crystalline solid and the NMR spectra did not show any starting material or major impurities. The NMR and IR spectra (available as Supporting Information) corresponded to literature $[3,40]$. We conclude that the purified product should in principle be clean enough for further use in synthetic applications.

\section{Possible extensions}

The experiment provided in this paper is a basic version that can be conducted in a single day of experimentation in the lab and requires about a day in total for preparation beforehand and data analysis and completion of lab notebook recording afterwards. There are ample opportunities for extension or adaptation to suit the personal preferences of teachers and / or students. Some options are:

- Start with a demonstration experiment: when some droplets of the TFAA solution are added to the mixture of $\mathbf{1}$ and DMSO, it turns red. This is an interesting experimental observation: apparently, the intermediate that is formed before adding base already has the typical color of the final product! After 1 or $2 \mathrm{~s}$, the color is gone again. Carrying this out in continuous-flow allows for adding the base exactly at the 'temporal sweet spot': the moment the maximum amount of intermediate is present.

Incidentally, this is an observation that any chemist should appreciate since it can, in principle, provide important clues as to the molecular structure of the intermediate that, due to its ephemeral nature, would have been easily missed by any other means of observation other than seeing it with the naked eye.

As such, in the context of chemical education, it can even be used to teach undergraduate students how to observe properly, how to take notes of observations in their laboratory notebook and to think about the meaning of observations and scientific evidence in general.

- Have students synthesize their own hydrazone, either benzophenone or an alternative, such as the ones mentioned by Javed and Brewer [3, 4] according to procedures such as described in [41, 42]. In our lab, one couple of students have successfully synthesized acetophenone hydrazone and subsequently observed at least partial successful conversion into the corresponding methylphenyldiazomethane ((1-diazoethyl)benzene).

- Have students try to determine the absolute yield of the diazo compound without the need for purification. Apart from losses due to purification itself, the pure compounds are unstable and may present safety problems. Determining the yield in another manner is not trivial [3, 39]; standard options are measuring the nitrogen evolution upon the addition of acid or quantifying a derivative such as an ester that can be formed upon the addition of a 
carboxylic acid. All of these methods are believed to underestimate the actual yield. Students could think about alternative manners, e.g. a spectroscopic quantification (UV-Vis, qNMR, IR) or a titration for instance.

- Have students try to use the diazo compound in a subsequent reaction. The conversion into an ester was already mentioned; similarly, an ether can be produced by reaction of $\mathbf{2}$ with an alcohol. But diazo chemistry is quite versatile [5], including the formation of (substituted) olefins, cyclopropanation of olefins, insertion in $\mathrm{O}-\mathrm{H}, \mathrm{N}-\mathrm{H}$ and $\mathrm{C}-\mathrm{H}$ bonds, the formation of carbonyl and sulfur ylides, 1,3-dipolar cycloadditions and even the production of highly substituted polymethylenes [6]

- Have students adapt the flow setup. The use of an extra syringe, pump and T-piece or Y-piece, enables the use of separate solutions of DMSO and $\mathbf{1}$ and, hence, removal of the confounding and even separate optimization of their stoichiometry. Another option would be to try the setup from [26] in which the substrate - an alcohol in that case is only added after the activated DMSO is produced. Students can investigate the influence this has on the yield of the reaction.

- Have students choose another experimental design, such as a Plackett-Burman design, the more advanced D-optimal, M-optimal or A-optimal designs or - the favorite amongst our students - a central composite design [37, 38]. Instead of these so-called response-surface methodologies, another option would be to use another optimization approach altogether, such as simplex optimization [37, 43]. Enthusiastic students could even program their own version of this algorithm.

- Have students think of different optimization criteria to use: do they want to maximize the conversion rate, the production per unit time or minimize the costs or the waste produced?

- If reactors of different internal volume are available, students could be challenged to try and scale up the reaction.

- Finally, if students and / or teachers observe the same problem that we describe in reference 36 , a challenging option might be to try and find the cause of the infrequent failure.

\section{Experimental}

\section{Determination of relative conversions}

Microreactor setup All flow chemistry experiments were conducted using a FutureChemistry b330 FlowStart education setup, consisting of three syringe pumps, a temperature controller and a microreactor holder equipped with a Peltier element. The microreactor was a FutureChemistry 'Short
Quench' microreactor consisting of borosilicate glass, with an internal volume (measured between the point where solutions 1 and 2 are mixed and the point where solution 3 is added) of $1 \mu \mathrm{L}$. The syringes used for dispensing the reactant solutions were two gas-tight $5 \mathrm{~mL}$ borosilicate glass syringes and one gas-tight $1 \mathrm{~mL}$ borosilicate glass syringe (Integrated Lab Solutions $\mathrm{GmbH}$ ). Connections between syringes and the microreactor consisted of FEP tubing, 1/16" O.D., $0.50 \mathrm{~mm}$ I.D. (IDEX art. Nr. 1548 L).

All experiments were conducted with solutions in dichloromethane (Fisher Scientific, purity $\geq 99 \%$ ) made from the chemicals as obtained from the manufacturers without further purification or drying: trifluoroacetic acid (Alfa Aesar, 299\%): $0.4 \mathrm{M}$; dimethyl sulfoxide (VWR Chemicals, technical grade): $0.6 \mathrm{M}$, together with benzophenone hydrazone (Acros Organics, purity $\geq 98 \%$ ): $0.2 \mathrm{M} ; N, N$ diisopropylethylamine (Fisher Scientific, purity $\geq 98 \%$ ): $1.435 \mathrm{M}$. The solution of dimethyl sulfoxide and benzophenone hydrazone was freshly made on a daily basis, since after a day of standing it became slightly opaque and a watersoluble precipitate formed at the bottom of the flask.

UV-analysis All quantitative Vis spectroscopic measurements for determination of relative conversions were performed offline on a Shimadzu UV-1800 UV-Vis spectrophotometer using a semi-micro quartz cuvette with a pathlength of $1 \mathrm{~cm}$.

\section{Purification trial}

For the 'purification trial' of $\mathbf{2}$, fresh bottles of the same chemicals were used, with the exception of: dimethyl sulfoxide (Acros Organics, purity $\geq 99.7 \%$, 'Extra Dry') and benzophenone hydrazone, which was recrystallized from a minimum volume of hot ethanol and dried in vacuo at $35^{\circ} \mathrm{C}$.

To the collected product solution in dichloromethane (approximately $20 \mathrm{~mL}$ ) an equal volume of pentane was added. The resulting organic phase was subsequently washed with saturated sodium bicarbonate solution $(1 \times 20 \mathrm{~mL})$, water $(3 \times 20 \mathrm{~mL})$ and brine $(2 \times 20 \mathrm{~mL})$, and dried over sodium sulfate. The solvent was removed in vacuo at room temperature. The residual dark purple-red oil was dissolved in a minimal amount of pentane and filtered over basic alumina (approx. $3 \mathrm{~cm}$ ). The solids were rinsed with pentane until the filtrate was colorless. The filtrate was evaporated in vacuo at room temperature, yielding the product as a dark purple-red crystalline solid.

All NMR spectra were collected on a Bruker $300 \mathrm{MHz}$ Avance III HD spectrometer equipped with a $\mathrm{BBO}$ probe at $298 \mathrm{~K}$ with a ${ }^{1} \mathrm{H}$ frequency of $300.13 \mathrm{MHz}$ and a ${ }^{13} \mathrm{C}$ frequency of $75.48 \mathrm{MHz}$. Tetramethylsilane $(\delta=0.00 \mathrm{ppm})$ or the residual protons of the deuterated solvent were used as internal references. ${ }^{1} \mathrm{H}^{13}{ }^{13} \mathrm{C}$ HSQC spectra were acquired using a $2870.6 \mathrm{~Hz}$ spectral width in F2 and 12,500 Hz spectral width 
in F1 using $512 \times 256$ points and processed to $512 \times 512$ points, 4 scans per increment, relaxation delay of $1.5 \mathrm{~s}$ and 1-bond $\mathrm{JCH}=145 \mathrm{~Hz} .{ }^{1} \mathrm{H}-{ }^{13} \mathrm{C} \mathrm{HMBC}$ spectra were acquired using a $2873.6 \mathrm{~Hz}$ spectral width in $\mathrm{F} 2$ and $16,778.5 \mathrm{~Hz}$ spectral width in F1 using $512 \times 128$ points and processed to $512 \times 512$ points, 8 scans per increment, a relaxation delay of $1.5 \mathrm{~s}$ and long-range $\mathrm{JCH}=8 \mathrm{~Hz}$.

${ }^{1} \mathrm{H}$ NMR $\left(\mathrm{CD}_{2} \mathrm{Cl}_{2}, 300 \mathrm{MHz}\right): \delta_{\mathrm{H}} 7.45-7.37$ (4H, m, H3, H5, H10 and H12), 7.35-7.29 (4H, m, H2, H6, H9 and H13), 7.24-7.17 (2H, m, H4 and $\mathrm{H} 11) .{ }^{13} \mathrm{C}\left\{{ }^{1} \mathrm{H}\right\}$ NMR $\left(\mathrm{CD}_{2} \mathrm{Cl}_{2}\right.$, $75 \mathrm{MHz}): \delta_{\mathrm{C}} 130.0$ (s, C1 and C8), 129.5 (s, C3, C5, C10 and C12), 126.0 (s, C4 and C11), 125.5 (s, C2, C6, C9 and C13), 62.7 (s, C7).). FT-IR (diamond ATR): 3057w (br, aromatic C-H), 3030w (br, aromatic C-H), 2032s (diazomethane N-N stretch), $1592 m$ (aromatic C-C), $1492 m$ (C-C), 1262w (C-N), 1075w, 1030w, $747 s, 688 s, 649 m, 472 m$

\section{Safety}

All experiments should be conducted in a fume hood. All chemicals should be treated with normal precaution: we teach our students to look up the safety information for all chemicals and products in the appropriate MSDSs themselves. They also have to come up with ways to quench and dispose any resulting reaction mixtures, products, solvents, etc. This information is discussed with a teacher or TA prior to performing the experiment.

For this experiment in particular, note that DMSO increases uptake of other chemicals through the skin. The produced diphenyldiazomethane is unstable and, like all diazo compounds, toxic and potentially explosive in purified form; complete purification is not recommended for undergraduate students. The produced diazo compound can be quenched using small amounts of dilute acetic acid or another dilute acid.

Consider having students wear appropriate gloves when filling, attaching or detaching syringes. To take up liquids in the syringes used for dispensing the liquid in the experiment, we used blunt needles (Sterican MIX $1.20 \times 40 \mathrm{~mm}$, B. Braun Melsungen AG).

\section{Conclusion}

We have presented a challenging flow chemistry experiment involving the synthesis of diphenyldiazomethane. Readers of this journal will no doubt agree that it is important for chemistry students to become acquainted with flow chemistry in their education. The presented experiment is suitable for this goal, but also provides an opportunity for activating and applying prior knowledge about various other chemical subjects. An important reason for this is the fact that multiple flow chemistry experiments can be performed in a limited amount of time. To paraphrase (1): in fact, more generally, chemical educators should realize that whenever they would want to teach techniques or concepts that benefit from repeatedly carrying out the same reaction (e.g. optimization, determining the scope or the kinetics of a reaction), it is advantageous to conduct the reaction in flow, not only due to the superior control over the reaction conditions and the resulting reproducibility of the process, but mainly due to the fact that multiple experiments can easily be conducted within the limited time frame of lab courses.

Acknowledgments Dr. Daniel Blanco-Ania and Koen van Asseldonk are acknowledged for helpful discussions. All undergraduate students who have carried out the experiment over the years are acknowledged for critical comments that contributed to the improvement of the experiment.

Data availability Measured data and spectra available from Supporting Information.

\section{Compliance with ethical standards}

Conflicts of interest On behalf of all authors, the corresponding author states that there is no conflict of interest.

Code availability Matlab code for generating Fig. 5 available from Supporting Information.

Open Access This article is licensed under a Creative Commons Attribution 4.0 International License, which permits use, sharing, adaptation, distribution and reproduction in any medium or format, as long as you give appropriate credit to the original author(s) and the source, provide a link to the Creative Commons licence, and indicate if changes were made. The images or other third party material in this article are included in the article's Creative Commons licence, unless indicated otherwise in a credit line to the material. If material is not included in the article's Creative Commons licence and your intended use is not permitted by statutory regulation or exceeds the permitted use, you will need to obtain permission directly from the copyright holder. To view a copy of this licence, visit http://creativecommons.org/licenses/by/4.0/.

\section{References}

1. Blanco-Ania D, Rutjes FPJT (2017) Continuous-flow chemistry in chemical education. J Flow Chem 7(3-4):157-158

2. Nieuwland PJ, Koch K, van Harskamp N, Wehrens R, van Hest JCM, Rutjes FPJT (2010) Flash chemistry extensively optimized: high-temperature Swern-Moffatt oxidation in an automated microreactor platform. Chem-Asian J 5(4):799-805

3. Javed MI, Brewer M (2007) Diazo preparation via dehydrogenation of hydrazones with "activated" DMSO. Org Lett 9(9):1789-1792

4. Javed MI, Brewer M (2008) Diphenyldiazomethane. Org Synth 85: 189-195

5. Maas G (2009) New syntheses of Diazo compounds. Angew Chem Int Edit 48(44):8186-8195

6. Hetterscheid DGH, Hendriksen C, Dzik WI, Smits JMM, van Eck ERH, Rowan AE et al (2006) Rhodium-mediated stereoselective polymerization of "carbenes". J Am Chem Soc 128(30):9746-9752

7. Delville MME, van Hest JCM, Rutjes FPJT (2013) Ethyl diazoacetate synthesis in flow. Beilstein J Org Chem 9:1813-1818 
8. Dommerholt J, Schmidt S, Temming R, Hendriks LJA, Rutjes FPJT, van Hest JCM et al (2010) Readily accessible Bicyclononynes for bioorthogonal labeling and three-dimensional imaging of living cells. Angew Chem Int Ed 49(49):9422-9425

9. Or 'synthesis', but that term may be somewhat confusing in the context of a chemistry paper.

10. Bergendahl C, Tibell L (2005) Boosting complex learning by strategic assessment and course design. J Chem Educ 82(4):645-651

11. Shiland TW (1999) Constructivism: the implications for laboratory work. J Chem Educ 76(1):107-109

12. Kornblum N, Powers JW, Anderson GJ, Jones WJ, Larson HO, Levand O, et al. (1957) A new and selective method of oxidation. J am Chem Soc 79(24):6562-

13. Kornblum N, Jones WJ, Anderson GJ (1959) A new and selective method of oxidation - the conversion of alkyl halides and alkyl Tosylates to aldehydes. J Am Chem Soc 81(15):4113-4114

14. Pfitzner KE, Moffatt JG (1963) A new and selective oxidation of alcohols. J Am Chem Soc 85(19):3027-3028

15. Pfitzner KE, Moffatt JG (1965) Sulfoxide-Carbodiimide reactions. I A Facile Oxidation of Alcohols. J Am Chem Soc 87(24):56615670

16. Pfitzner KE, Moffatt JG (1965) Sulfoxide-Carbodiimide reactions. II Scope of the Oxidation Reaction. J Am Chem Soc 87(24):5670 5678

17. Fenselau AH, Moffatt JG (1966) Sulfoxide-Carbodiimide reactions. III Mechanism of the Oxidation Reaction. J Am Chem Soc 88(8):1762-1765

18. Tojo J, Fernández M In: Tojo J, editor. (2006) Oxidation of alcohols to aldehydes and ketones - a guide to current common practice. New York: Springer Science+Business Media, Inc.

19. Mancuso AJ, Huang SL, Swern D (1978) Oxidation of long-chain and related alcohols to carbonyls by dimethyl-Sulfoxide activated by Oxalyl chloride. J Org Chem 43(12):2480-2482

20. Omura K, Swern D (1978) Oxidation of alcohols by activated dimethyl-Sulfoxide - preparative, steric and mechanistic study. Tetrahedron 34(11):1651-1660

21. Omura K, Sharma AK, Swern D (1976) Dimethyl SulfoxideTrifluoroacetic anhydride - new reagent for oxidation of alcohols to carbonyls. J Org Chem 41(6):957-962

22. Huang SL, Omura K, Swern D (1976) Oxidation of Sterically hindered alcohols to carbonyls with dimethyl SulfoxideTrifluoroacetic anhydride. J Org Chem 41(20):3329-3331

23. Albright JD, Goldman L (1965) Dimethyl Sulfoxide-acid anhydride mixtures. New reagents for oxidation of alcohols. J Am Chem Soc 87(18):4214-4216

24. Albright JD, Goldman L (1967) Dimethyl Sulfoxide-acid anhydride mixtures for oxidation of alcohols. J Am Chem Soc 89(10):2416 2423

25. Huang SL, Omura K, Swern D (1978) Further studies on oxidation of alcohols to carbonyl-compounds by dimethyl SulfoxideTrifluoroacetic anhydride. Synthesis-Stuttgart 1978(4):297-299

26. Kawaguchi T, Miyata H, Ataka K, Mae K, Yoshida J (2005) Roomtemperature Swern oxidations by using a microscale flow system. Angew Chem Int Edit 44(16):2413-2416

27. Van der Linden JJM, Hilberink PW, Kronenburg CMP, Kemperman GJ (2008) Investigation of the Moffatt-Swern oxidation in a continuous flow microreactor system. Org Process Res Dev 12(5):911-920
28. Keirs D, Overton K (1987) Conversion of amines into imines by Swern oxidation. J Chem Soc Chem Comm 1987(21):1660-1661

29. Jinbo Y, Kondo H, Taguchi M, Sakamoto F, Tsukamoto G (1994) Synthesis of new DNA Gyrase inhibitors - application of the DMSO oxidation to the conversion of the amine into the imine. J Org Chem 59(20):6057-6062

30. Gentilucci L, Grijzen Y, Thijs L, Zwanenburg B (1995) Convenient synthesis of optically-active 2h-Azirine-2-carboxylic esters by Swern oxidation of Aziridine-2-carboxylic esters. Tetrahedron Lett 36(26):4665-4668

31. Kamal A, Rao NV (1996) A new route for the synthesis of Pyrrolo[2,1-c][1,4]benzodiazepine antibiotics via oxidation of cyclic secondary amine. Chem Commun 1996(3):385-386

32. Torssell K (1966) Mechanisms of Dimethylsulfoxide oxidations. Tetrahedron Lett 7(37):4445-4451

33. Tran DN, Battilocchio C, Lou SB, Hawkins JM, Ley SV (2015) Flow chemistry as a discovery tool to access $\mathrm{sp}(2)-\mathrm{sp}(3)$ crosscoupling reactions via diazo compounds. Chem Sci 6(2):11201125

34. Movsisyan M, Delbeke EIP, Berton JKET, Battilocchio C, Ley SV, Stevens CV (2016) Taming hazardous chemistry by continuous flow technology. Chem Soc Rev 45(18):4892-4928

35. Hock KJ, Koenigs RM (2018) The generation of Diazo compounds in continuous-flow. Chem-Eur J 24(42):10571-10583

36. Of course, if students make a mistake in preparing their solutions, no red droplets will be visible. In the course of preparing this paper, we have noticed however, that in $\sim 1$ out of 10 cases for no apparent reason the reaction produces no product at all; preparing the $\mathbf{1} /$ DMSO solution anew in - as far as we are aware - exactly the same manner and restarting the reaction then results in product formation again. In our own experience with this particular experiment, a 10\% failure (a 90\% success rate, and with a simple remedy for the remaining 10\%: just make one or two stock solutions again or use someone else's) is not prohibitive at all, but sometimes just delays the commencement of the experiment proper, by $\sim 15 \mathrm{~min}$.)

37. Massart DL, Vandeginste BGM, Buydens LMC, De Jong S, Smeyers-Verbeke J (1997) Handbook of Chemometrics and Qualimetrics part a. Massart DL, editor

38. Leardi R (2009) Experimental Design in Chemistry: a tutorial. Anal Chim Acta 652(1-2):161-172

39. Andrews SD, Day AC, Raymond P, Whiting MC (1988) 2Diazopropane. Org Synth 50-9:392-394

40. Yates P, Shapiro BL (1957) Aliphatic Diazo compounds. 3. Infrared spectra. J Am Chem Soc 79(21):5756-5760

41. Patrick TB, Flory PA (1984) Direct fluorination of aryl ketone Hydrazones. J Fluor Chem 25(2):157-164

42. Holton TL, Shechter H (1995) Advantageous syntheses of Diazocompounds by oxidation of Hydrazones with Lead-Tetraacetate in basic environments. J Org Chem 60(15):4725-4729

43. Bezerra MA, dos Santos QO, Santos AG, Novaes CG, Ferreira SLC, de Souza VS (2016) Simplex optimization: a tutorial approach and recent applications in analytical chemistry. Microchem J 124:45-54

Publisher's note Springer Nature remains neutral with regard to jurisdictional claims in published maps and institutional affiliations. 